February 11, 2022

\section{2}

3

4

$5 \quad$ Leo Goldstein ${ }^{i}$

6

7

8

Leo Goldsteini Brooklyn, NYC.

\title{
Re-Analysis: New York City 2019 Measles Outbreak Happened in Sufficiently Vaccinated Population
}

\section{Summary}

The measles outbreak, which started in New York city in 2018, with most cases occurring February - May 2019, is usually described as the consequence of insufficient vaccination in the affected community, mainly among "ultra"-Orthodox Jews of Williamsburg and Borough Park,

However, the vaccination rates in this community were very high, and increased from 93.5-96\% in the yeshivas (Jewish religious schools) at the beginning of the outbreak, to $\sim 97-99 \%$ after the vaccination campaign in October - December of 2018. These vaccination rates were more than sufficient to quickly suppress an outbreak, especially when using basic countermeasures such as early isolation of the sick.

Re-analyzing the data, re-creating the timeline of the events, and comparing this outbreak with the past vaccination rates and outbreaks, leads to the conclusion: the outbreak's persistence and growth were driven by the orders and mandates imposed by the NYC Department of Health and Mental Hygiene (NYC DOHMH).

Possibly the most harmful DOHMH action was the exclusion order issued on December 6, 2018, excluding all unvaccinated children from all yeshivas, even in the absence of measles cases. This order effectively concentrated unvaccinated children together, defeating herd immunity.

Unvaccinated children had to spend an unusually large fraction of daytime at home. Excluded kids with measles infected infants, who were too young for vaccination.

Additionally, immunoglobulin (IG), a highly effective post-exposure prophylaxis, was removed from recommendations for the affected communities, perhaps to encourage additional vaccination. Some documents recommended IG only for infants below six months.

The definition of "vaccinated" changed in February - April 2019, when NYC DOHMH mandated an additional MMR vaccination for infants (6-11 months) in the affected communities, contrary to accepted scientific findings.

iah@defyccc.com 
32 The discontent with these heavy-handed mandates and other harassment were mistaken for

33 anti-vaccination attitude and blamed on anti-vax propaganda and "misinformation".

34 Comparing the government's reactions during this measles outbreak to their reactions during 35 the beginning of the COVID-19 outbreak, we find some parallels. Many of the same NYC 36 government officials, who mishandled the measles outbreak in 2018-19, also handled the 37 response to the COVID-19 pandemic in 2020. New York became the COVID-19 epicenter in 382020.

39 Some of the same harmful measures, practiced by the NYC DOHMH during the measles 40 outbreak, were also implemented by it and CDC during the COVID-19 crisis, including heavy-

41 handed mandates, over-emphasis on vaccination, and withdrawal of post-exposure prophylaxis 42 and treatment. 


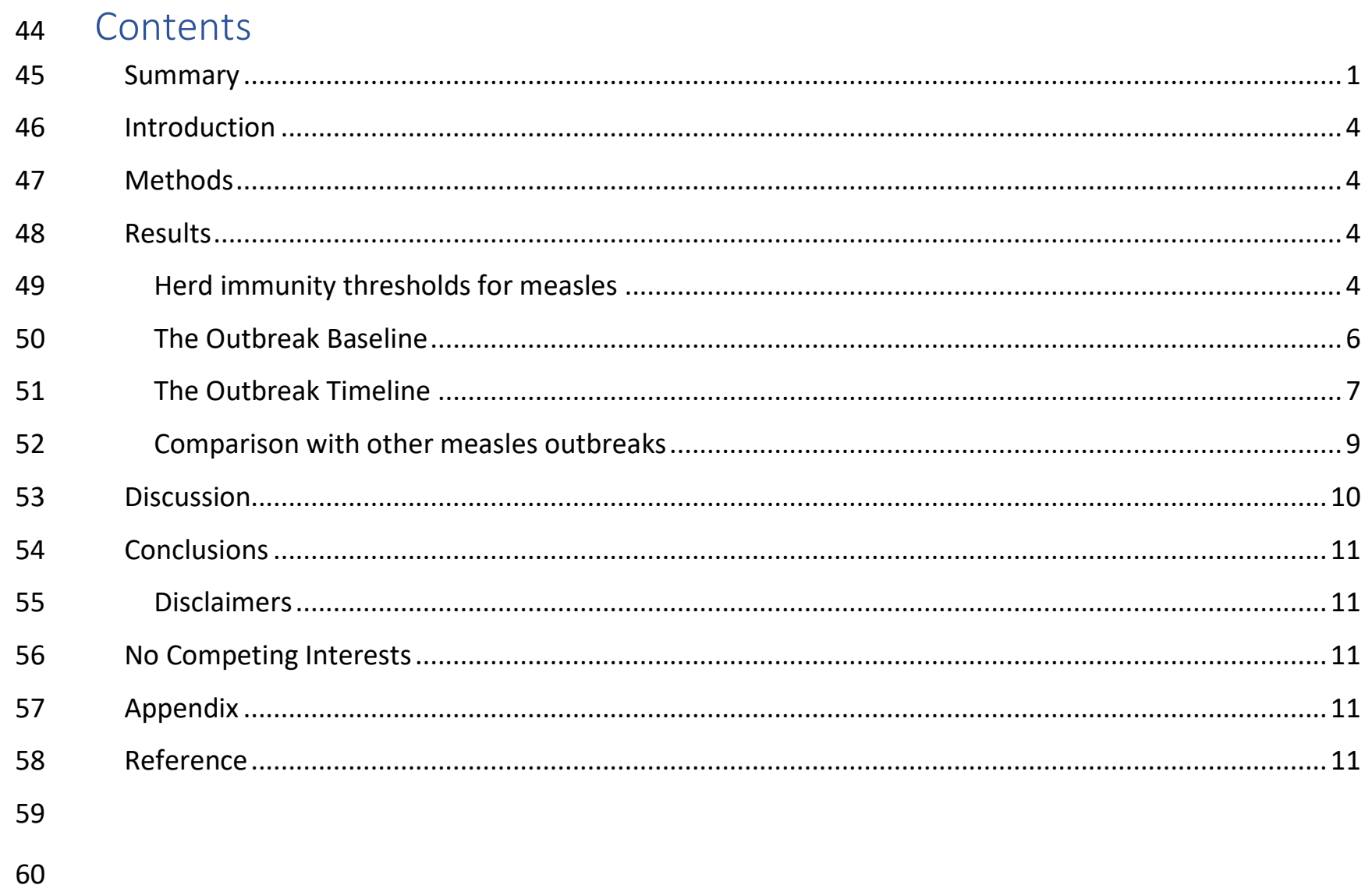


62 There were 10 million cases worldwide in $2018^{1}$. In 2000, measles was declared eliminated in the US. This means that there has been no continuous presence of measles, but small outbreaks caused by imported cases occur annually. 2019 was a record year, with a total of 1,282 cases ${ }^{2}$. Measles reporting is mandatory, so the statistics is readily available. Measles is considered eliminated throughout Americas.

The measles virus is transmitted through aerosols and is very contagious. After an incubation period, early symptoms (fever, cough, coryza-running nose, and conjunctivitis) appear. A characteristic rash appears about four days later ${ }^{34}$. Early symptoms can easily be mistaken for other diseases, including common cold and flu ${ }^{6}$. The rash typically appears 14 days after infection, but that can vary from 7 to 21 days, usually faster in children than in adults ${ }^{5}$. Measles is most contagious from the appearance of early symptom to the onset of the rash, although transmission can happen as early as 1-2 days before the early symptoms. The mortality from measles is very low.

Most cases during the 2018-19 measles epidemic in New York occurred among the Jewish population in three areas: Williamsburg, Borough Park (both Brooklyn, NYC), and Rockland County, just outside of NYC. These areas are not adjacent to each other. The Jewish population is a large minority in each area and belongs to a closely-knit "ultra-Orthodox"/Orthodox community. Rockland County is not included in this analysis because it is outside of NYC and had a relatively small number of cases. There, the Jewish "ultra-Orthodox"/Orthodox communities of Williamsburg and Borough Parks are referred to as "the affected community." The affected community was more susceptible to measles transmission than the average population due to the prevalence of large families with many children.

\section{Methods}

83 This is a historical re-analysis of the data from the NYC 2018-19 measles outbreak, using government provided statistics, public health orders, news sources from that period, and medical/epidemiological papers.

\section{Results}

Herd immunity thresholds for measles

HIT with and without countermeasures

Herd immunity threshold (HIT) is the fraction of the population that must become immune to an infectious disease to prevent the development or growth of an outbreak. Statistically, HIT depends on the pathogen, the conditions, and the community response. Based on the assumed $R_{0}=14$ in the average conditions, measles' HIT $=1-1 / 14=93 \%$. Achieving that would require $95 \%$ vaccination coverage with two doses of MMR vaccines, accounting for a vaccine failure rate of $\sim 3 \%$ (after two doses).

95 However, simple countermeasures can drastically decrease the required immunity threshold and contain and suppress outbreaks within areas with a much lower population immunity. The drop in measles cases in 1970s and 1980s occurred despite the decrease in the population's natural immunity levels caused by the effective vaccine with insufficient coverage. 
Early measles symptoms match many other childhood diseases, so measles cannot be diagnosed timely in normal settings. But once a measles outbreak is recognized, measles cases can be detected and isolated very early. Parents can be trusted to watch for early symptoms and isolate their child upon suspicion, especially within tightly knit communities. This measure alone can decrease transmission by an order of magnitude.

Additionally, immunoglobulin (IG) is available and is highly effective in preventing the disease and further transmission, if administered within six days after exposure (post-exposure prophylaxis or PEP). The MMR vaccine can be also used as PEP, but only within three days of exposure, which may be too short time. A 2013 clinical trial in NYC found that IG PEP had $100 \%$ effectiveness compared to $84 \%$ effectiveness of the MMR PEP ${ }^{7}$.

Thus, the HIT required to suppress a known outbreak is much lower than the HIT required to prevent outbreaks.

\section{Recent history of population immunity}

112 Before the introduction of the measles vaccination in 1963, herd immunity levels were achieved by

113 natural infection and consequent immunity ${ }^{8}$. After the introduction of the vaccine, natural

114 immunization rates dropped, and became insignificant since 1967.

115 Before 1963, US had about 500,000 reported cases annually, and an estimated 3-4 million cases in total 1169. In 1967, annual reported cases dropped to around 100,000. In 1981-1988 there were average 3,000 117 annual cases (ref. ${ }^{10}$, Fig. 33; ref. ${ }^{11}$ ). Per 100k people, annual reported cases dropped from an average of $\sim 300$ (before 1960) to below 40 (by 1967 and later) ${ }^{12}$. The reporting has also improved.

In the 1972-1988 period, the US average immunization rate for pre-school children (19-35 months) was between $60 \%$ and $70 \%$, by a single dose of the measles vaccine (ref. ${ }^{11}$, the Figure; ref. ${ }^{13}$, Fig. 1 ). With additional vaccinations at an older age, an immunity rate of $81 \%$ in 21 -year-olds was achieved

122 (measured by serological testing in $1988-94^{8}$ ). In 1977 , only $58 \%$ of $15-19$-year-olds in Nassau County, 123 NY, had immunity to measles ${ }^{14}$.

124 The coverage was unequally distributed, with more vulnerable communities having lower vaccination 125 rates. Vulnerability to measles is associated with more crowded living quarters, with children of 126 different ages in close contact. Thus, the vaccine coverage in vulnerable communities in late 1970s and 127 early 1980 s was probably $<50 \%$ at 24 months and $<60 \%$ in schools. Measles outbreaks occurred but 128 were quickly put out with significantly less resources than exist today ii.

129 Currently, in the US, $90 \%$ of children are vaccinated against measles by 24 months ${ }^{15}$, very uniformly by 130 demographic (88\%-92\%), but variable by geography. Many areas have a vaccination coverage of less 131 than $80 \%$ (WSJ ref. ${ }^{16}$ and interactive map ${ }^{17}$ ).

\footnotetext{
ii This is not encouragement of decreasing MMR vaccination coverage now. Prevention is better than remediation. Also, there is much more international travel, causing more imported measles cases. We are likely not aware of them because at the current population immunity levels they do not cause outbreaks. Thus, the author concurs with the current policy of MMR immunization having goal of achieving $95 \%$ coverage in each school and location and maintaining the measles elimination status.
} 
Infant vaccination

Studies have shown that measles vaccination before 12 months has low immunogenicity and might cause failure to acquire immunity even after multiple repeated vaccination at older age ${ }^{18}$. Vaccinating 6 month-old babies against measles produces no immunity in $64 \%$ cases, even if the baby has not received antibodies from the mother ${ }^{19}$. Vaccination attempts at 6-11 months, even if successful, do not last long and are not entirely remediated by future vaccination ${ }^{20}$.

Vaccination rates and conditions during the NYC outbreak

\section{Schools' statistics}

The average vaccination rates in the affected community at the beginning of the outbreak were $96 \%$ among school- and daycare children, the same as in general private schools in Brooklyn. See Appendix, sheets S2 and S3. In the affected community's schools in the four zip codes singled out by the April 9 order, the vaccination rate was slightly lower at $93.5 \%$ (Appendix, S4). The vaccination rate in public schools was $99-100 \%$.

These vaccination rates in the affected community were higher than the national average, although not sufficient to prevent a measles outbreak in the existing conditions. But there were significant number of additional vaccinations in October and November $2018{ }^{21}$, probably raising vaccination rates to $97-99 \%$ (see below). More importantly, once a measles outbreak is recognized, it could be suppressed at much lower vaccination rate than existed at its start. This did not happen.

\section{Population statistics}

Population vaccination rates usually counted in children at 24 months or 19-35 months. The number is expected to be slightly lower than in schools. The NYC public health officials think they know these rates well, because NYC has a Citywide Immunization Registry, and healthcare providers are obligated to report all vaccinations to the NYC government ${ }^{21}$. Ref. ${ }^{21}$ contains average rate of measles vaccinations in Williamsburg, including but not limited to the affected community, for the ages bracket 12 to 59 months. It was $91.1 \%$ in the end of the pandemic, probably achieved by the end of 2018 . It is likely underestimate because it does not count vaccinations made outside of the NYC. The recommended vaccination age is $12-15$ months. We can assume that the vaccination rate in the affected community at 24 months was above $95 \%$ by the end of 2018 .

\section{The Outbreak Baseline}

There are no Jewish religious objections to vaccination. Even the New York Times agreed with that in $2015^{22}$.

"This week, in fact, the Orthodox Union, along with the Rabbinical Council of America, issued a statement that said that there are "halachic obligations to care for one's health as well as to take measures to prevent harm and illness to others, and Jewish law defers to medical experts in determining and prescribing appropriate medical responses to illness and prevention." The group concluded that the vaccination of children is "the only responsible course of action."'"

The religious leadership of the affected community took the 2018 measles outbreak very seriously from the beginning and strongly supported. It demanded from its members vaccination and adherence to city guidelines. From Hamodia, the leading "ultra"-Orthodox newspaper (November 13, 2018) ${ }^{23}$ : 
"Slowly but surely we're getting there [full MMR vaccination coverage]," the administrator of a cheder [equivalent of pre-school] who requested anonymity told Hamodia. "I called the two parents in the school who had exemptions and told them that we can't let their kids come to school until their get their shots. We're working with them and one already got the shots."

The administrator's experience is mirrored by several other reports that many parents who had previously claimed a "religious exemption" from state vaccination requirements had agreed to immunize their children under the threat of exclusion from schools.

According to reports, more than 2,500 individuals were vaccinated over the past two weeks"

See also "Chicago Rabbanim Address Measles Outbreak" (November 15, 2018) ${ }^{24}$, and others. In April 2019, even stronger language was used towards those community members who appeared to avoid vaccination ${ }^{25}$ : "Senseless! Heartless! Torah-less and Reckless"

This was not necessary, as almost all children in the affected community were vaccinated by that time. The deputy commissioner of NYC DOHMH later confirmed ${ }^{26}$ that "after the outbreak was announced, vaccination rates in Williamsburg rose from around 70 percent, to about 92 percent." The government documents and scientific literature cite higher initial vaccination rates than $70 \%$.

Satmar Hasids were the majority within the affected community. From Hamodia, June 2019 27:

"The United Talmudical Academy (UTA) yeshivah network, representing the Satmar community ... has nearly 11,000 students in 12 schools in Williamsburg. Around 400 had religious exemptions prior to the December [2018] order, according to administrator Rabbi Chaim Mandel. Rabbi Mandel says around 80 percent of these students got vaccinated following the order ..."

No prominent rabbi objected to vaccination, contrary to Internet rumors. If any "measles parties" took place, they were rare and were not a factor.

\section{The Outbreak Timeline}

This is a short timeline of Williamsburg and Borough Park, Brooklyn, NYC during the 2018-19 outbreak. Case times are determined by rash onset. Because almost all cases occurred in children, the most likely lag is 10-14 days, less likely lag is 7-9 and 15-17 days. In ref. ${ }^{21}$, Fig. 1 shows the number of new weekly cases in Williamsburg and Borough Park, Fig. 2 shows the number of weekly MMR vaccinations for ages 12-59 months in Williamsburg. The weekly new cases for NYC should also be available from ref. ${ }^{28}$ Williamsburg had a total of 473 cases, Borough Park had a total of 121 cases, other NYC areas had small numbers of cases. There was an importation of a few cases from abroad during the outbreak, but their impact was not significant compared with the hundreds of existing ones.

The outbreak in the affected community was connected to a smaller outbreak in Rockland County, just outside of NYC. Rockland county issued a school exclusion order for unvaccinated children on Dec $5{ }^{29}$ and an additional, unprecedented order excluding unvaccinated children from most public spaces on Mar $26^{30}$. The actions taken by NYC were similar to the actions of the Rockland County government, in approximately the same times. Thus, the impact of Rockland County does not distort the timeline below.

2018, Sep 30: A child with measles rash is identified within the affected community in NYC. 
Oct 18: After a few more cases, the NYC DOHMH declares measles outbreak ${ }^{31}$.

Oct-Nov: The affected community, alerted by the outbreak declaration, gets additional vaccines, elevating vaccination rates wherever they were insufficient.

Nov 6: National, state, and city elections occur. The outcome of these elections may have influenced the NYC government.

Dec 6: After less than 60 measles cases, NYC DOHMH issues a very unusual exclusion order. All unvaccinated and "under vaccinated" students in the affected community are excluded from yeshivas in about a dozen zip codes, even where no measles cases are found ${ }^{32}{ }^{21}$. Letters ${ }^{33}{ }^{34}$ sent to principals and parents stating:

"Effective Friday, December 7th, every student attending a yeshiva in the below zip codes in Borough Park and Williamsburg who is not vaccinated with the required number of doses of measles-mumps-rubella (MMR) vaccine will not be permitted to attend school, regardless of whether a case of measles has occurred in the school."

Compliance with this order varies. The measles incidence remains low ( 3 new cases/week) through December-January and then increases to 10-20 new cases/week in late January - February.

2019, Feb 28: NYC steps up enforcement of its exclusion order, and raises the agitation further ${ }^{35}$.

"Providers serving the affected Orthodox Jewish communities in Brooklyn should closely follow NYC Department of Health immunization and other guidance to accelerate the end of this local outbreak."

"During this outbreak, if parents of unvaccinated children in the affected community request titers to confirm immunity in order to be allowed to return to school where exclusions are in effect, providers should assume they likely had measles and should report the suspected case to the DOHMH"

"Last week, the Department expanded vaccination recommendations for providers serving the Orthodox Jewish community to include an early, extra dose of the MMR vaccine for children between the ages of 6 months to 11 months who live in Williamsburg and Borough Park."

Measles vaccination of 6-months-olds has been found ineffective and harmful; see above.

2019, March 14: Another announcement, indicating increased enforcement of the exclusion order, is issued ${ }^{36}$. Frequency of new measles cases continues to grow.

Mar 17 - Apr 6: Peak measles incidence, $>50$ new cases/week. This corresponds to increased spread following the Feb 28 announcement.

Apr 8-9: The heaviest intervention by NYC. De Blasio on April $8^{37}$ :

"Today we are declaring a public health emergency effective immediately. This will mandate vaccines for people living in the affected area."

NYC DOHMH press release ${ }^{38}$ : 
"The measles outbreak in the Orthodox Jewish community is now at 285 cases since it began last October."

NYC DOHMH Commissioner Order ${ }^{39}$ :

"IT IS HEREBY ORDERED that any person who lives, works or resides within the 11205, 11206, 11211 and/or 11249 zip codes and who has not received the MMR vaccine within forty eight (48) hours of this Order being signed by me shall be vaccinated against measles unless such person can demonstrate immunity to the disease or document to the satisfaction of the Department that he or she should be medically exempt from this requirement."

The order also demands vaccination of all babies, starting at six months (also within 48 hours), and threatens "violators" with prison ${ }^{40}$.

Apr 17: The Apr 9 order is replaced by the Board of Health resolution ${ }^{41}$. The language around vaccinating babies is clarified to mean "six months of age or older". The threat of imprisonment is removed. Having measles in the listed four zip codes is declared illegal ${ }^{42}$.

Apr 24: A new NYC order omits the highly effective IG treatment from post-exposure treatments in the affected community ${ }^{43}$ (compare to the earlier one ${ }^{44}$ ).

Apr 14 - May 4: undeterred by the NYC orders, measles cases continue, at 35 new cases per week. There might be underreporting, as the city made it illegal to have measles in the designated zip codes ${ }^{41}$.

May 5 - Jul 20: measles incidence drops. The last case is recorded in July.

As evidenced by the timeline, before NYC DOHMH issued any orders or mandates, measles cases were few (approximately 60 cases in 4 months) and vaccination rates went up in the affected community. So contrary to the later claims by NYC officials, very few helpful vaccine doses were administered after April 9. The 3,844 vaccines administered in Williamsburg ${ }^{45}$ to the general population included repeated vaccines in children who had already been immunized, and vaccines in infants (less than $1 \mathrm{y} / \mathrm{o}$ ), contrary to scientific recommendations and with no effect on transmission.

NYC DOHMH excessive exclusion orders drove the outbreak to continue spreading and disproportionately affect infants. By excluding unvaccinated children from schools and pre-schools, NYC DOHMH forced those unvaccinated children to concentrate in other spaces, mostly homes. Since the population immunity in those few unvaccinated children was almost zero, measles could easily spread amongst them. Since they were mostly at home, they also infected infants between 6 and 12 months. This accounts for the increase in cases and the unusually high rate of infected infants.

Some outbreak-related NYC DOHMH orders ${ }^{46} 47$ are not included here because of low relevance.

\section{Comparison with other measles outbreaks}

Amish, 2014

The 2014 measles outbreak among Ohio Amish had 383 cases out of an Amish population of 32,630. In the past, the Amish strongly objected to vaccines, but by 2014 , an estimated $40 \%-50 \%$ of their children were vaccinated ${ }^{48}$. The immunity rate of adults (18-40) was much lower, leading to unusually high rates of infection in this age group, and transmission from adults to children. This outbreak matches the 
expectation for a low vaccination rate. While the Amish traditionally have multiple children, their rural lifestyle is likely associated with lower HIT. The outbreak was controlled with the help of MMR vaccination, covering an additional $\sim 27 \%$ of the community. No IG PEP was administered. In 2017, there was a small outbreak in a Somali-American community of Hennepin County, Minnesota ${ }^{49}$. There, children were vaccinated relatively late. Only $36 \%$ of 2 -years-olds were vaccinated at the time of the outbreak ${ }^{50}$. The vaccination levels slowly increased with the age but remained insufficient. These Somali Americans also have large families with many children. This low level of vaccination among kids is expected to lead to outbreaks. Yet only 75 cases occurred in the 2017 outbreak, including cases outside of the Somali-Minnesotan community. IG PEP was administered ${ }^{51}$.

Israel, 2018-19

A larger Israeli outbreak of 2018-19 led to the importation of cases into NYC \& NYS. The outbreak was centered in the "ultra-Orthodox" Jewish population in Jerusalem ${ }^{52}$. There were 2,202 total cases in the Jerusalem district, mostly among 220,000 "ultra-Orthodox" community members. The child vaccination rate of $78 \%$ in that population was clearly insufficient for the living conditions there - large families living in a very high population density. It is unclear if any vaccination rate would be sufficient to prevent a measles outbreak in such conditions. The affected community of NYC had a much lower population density.

\section{Discussion}

301

302

303

304

305

The 2018-19 NYC measles epidemic lasted a long time not due to low vaccination rates, but because of excessive and unhelpful government intervention. The single worst action was probably the exclusion order, concentrating unvaccinated and infected children together, mostly in homes, where infants were also easily exposed. Additionally, the government removed a highly effective treatment (IG) from the affected communities.

These and other governmental measures burdened the parents. The resources they needed to protect unvaccinated children and infants from potentially exposed and infectious brothers and sisters, were instead spent complying with heavy-handed city orders.

Removing unvaccinated children from schools during an outbreak is not uncommon and is often voluntarily done by the parents and educators working together, in the specific school where cases are present. Blanket exclusion orders have just removed susceptible children from schools, where they were protected by the herd immunity, to spaces which became pockets of infection.

\section{"Anti-vax" groups}

There is always a small percentage (1-3\%) of parents who refuse vaccination because of personal beliefs, concerns, or a child's medical conditions not recognized for medical exemptions. There are also vaccine failures. Even combining both factors would not sustain a measles outbreak in a highly vaccinated population once the outbreak is detected and simple countermeasures are applied.

Reports show that significant outside "anti-vax" groups, such as Kennedy Jr.'s "Children Health Defense" appeared on the scene only after the April 9, 2019 Order, as evidenced in this lawsuit ${ }^{53}$. These groups became involved too late to persuade the local population and could not have had a significant impact. Anyway, most children had already been vaccinated. 


\section{Perceived resistance to vaccination}

In March-April 2019, NYC and Rockland County officials started complaining about insufficient cooperation from some members of the affected communities. These complaints came only after the government had unnecessarily harassed them. For example, they made six rounds of robocalls to 30,000 families each. Most of these families had already vaccinated all of their children ${ }^{45}$. Then government officials acted surprised that some residents refused taking their phone calls.

Naturally, sudden changes to the vaccination schedule, harassment, and coercion caused mistrust, suspicion, and reduced cooperation by the community members.

\section{Conclusions}

The affected Orthodox Jewish community in NYC had a high measles vaccination rate, sufficient to stop the outbreak without government intervention. However, NYC DOHMH activism, especially exclusion orders, fanned up the outbreak. NYC DOHMH made an excessive emphasis on vaccination, at the expense of IG and non-pharmacological countermeasures.

One lesson that was apparently not learned is to avoid becoming obsessed with one mild disease. Today, measles is a mild disease with low rates of complications. While avoiding measles is a worthy goal, an outbreak with a few dozens of cases should not turn into an obsession.

\section{Disclaimers}

The author supports the current policy of measles elimination by vaccination with MMR/MMRV.

The author did not use and did not have access to non-public sources in preparing this paper.

\section{No Competing Interests}

The author declares no competing interest. No funding was provided for this work.

\section{Appendix}

The Appendix is available with this manuscript. It might be also available from https://defyccc.com/data/Measles-Outbreak-NYC-2019-ReAnalysis-Appendix.xlsx

The Appendix is based on ref. ${ }^{54}$

\section{Reference}

1. W.H.O. More than 140,000 die from measles as cases surge worldwide. Published 2019. Accessed February 1, 2022. https://www.who.int/news/item/05-12-2019-more-than-140-000-die-frommeasles-as-cases-surge-worldwide

2. CDC. Measles Cases and Outbreaks. Centers for Disease Control and Prevention. Published January 4, 2022. Accessed February 1, 2022. https://www.cdc.gov/measles/cases-outbreaks.html

3. Gastanaduy PA, et al. Measles. In: VPD Surveillance Manual. ; 2018. https://www.cdc.gov/vaccines/pubs/surv-manual/chpt07-measles.pdf 
4. CDC. Measles Signs and Symptoms. Centers for Disease Control and Prevention. Published November 5, 2020. Accessed February 1, 2022. https://www.cdc.gov/measles/symptoms/signssymptoms.html

5. Louisiana Public Health. MeaslesManual.pdf. Published online June 18, 2012. Accessed February 1, 2022. https://ldh.la.gov/assets/oph/Center-PHCH/Center-CH/infectiousepi/EpiManual/MeaslesManual.pdf

6. Koenig K, Alassaf W, Burns M. Identify-Isolate-Inform: A Tool for Initial Detection and Management of Measles Patients in the Emergency Department. WestJEM. 2015;16(2):212-219. doi:10.5811/westjem.2015.3.25678

7. Arciuolo RJ, Jablonski RR, Zucker JR, Rosen JB. Effectiveness of Measles Vaccination and Immune Globulin Post-Exposure Prophylaxis in an Outbreak Setting-New York City, 2013. Clinical Infectious Diseases. 2017;65(11):1843-1847. doi:10.1093/cid/cix639

8. Hutchins SS, Redd SC, Schrag S, et al. National serologic survey of measles immunity among persons 6 years of age or older, 1988-1994. MedGenMed. Published online January 24, 2001:E5. https://pubmed.ncbi.nlm.nih.gov/11320344/

9. CDC. About Measles Vaccination | CDC. Published March 26, 2021. Accessed February 7, 2022. https://www.cdc.gov/vaccines/vpd/measles/index.html

10. CDC MMWR. MMWR Summary of Notifiable Diseases, United States, 1993. Published 1993. Accessed February 1, 2022. https://www.cdc.gov/mmwr/preview/mmwrhtml/00035381.htm

11. CDC MMWR, Whitney CG, et al. Benefits from Immunization During the Vaccines for Children Program Era - United States, 1994-2013. Published online 2014. Accessed February 1, 2022. https://www.cdc.gov/mmwr/preview/mmwrhtml/mm6316a4.htm

12. Our World in Data. Rate of reported cases and deaths from measles in the USA. Our World in Data. Published February 1, 2022. Accessed February 1, 2022. https://ourworldindata.org/grapher/measles-cases-and-death-rate

13. CDC. Vaccine-Preventable Diseases, Immunizations, and MMWR --- 1961--2011. Published 2011. Accessed February 10, 2022. https://www.cdc.gov/mmwr/preview/mmwrhtml/su6004a9.htm

14. Brookman RR, Rauh JL. Immunization of Adolescents. Pediatric Annals. Published online September 1978. Accessed February 10, 2022. https://www.proquest.com/docview/1866018566/citation/2F8EB2527B124974PQ/1

15. CDC Data. Health, United States 2019, Table 31. Published online 2019. https://www.cdc.gov/nchs/data/hus/2019/031-508.pdf

16. Moriarty BA Taylor Umlauf and Dylan. Thousands of Schools Fall Below Recommended Measles Vaccination Rate. Wall Street Journal. https://www.wsj.com/articles/thousands-of-schools-fallbelow-recommended-measles-vaccination-rate-11570122220. Published October 3, 2019. Accessed February 1, 2022. 
17. Graphics WSJ. What's the Measles Vaccination Rate at Your Child's School? WSJ. Published 2019. Accessed February 1, 2022. https://www.wsj.com/graphics/school-measles-rate-map/

18. Carazo S, Billard MN, Boutin A, De Serres G. Effect of age at vaccination on the measles vaccine effectiveness and immunogenicity: systematic review and meta-analysis. BMC Infectious Diseases. 2020;20(1):251. doi:10.1186/s12879-020-4870-x

19. Gans HA. Deficiency of the Humoral Immune Response to Measles Vaccine in Infants Immunized at Age 6 Months. JAMA. 1998;280(6):527. doi:10.1001/iama.280.6.527

20. Brinkman ID, de Wit J, Smits GP, et al. Early Measles Vaccination During an Outbreak in the Netherlands: Short-Term and Long-Term Decreases in Antibody Responses Among Children Vaccinated Before 12 Months of Age. The Journal of Infectious Diseases. 2019;220(4):594-602. $\underline{\text { doi:10.1093/infdis/iiz159 }}$

21. Zucker JR, Rosen JB, Iwamoto M, et al. Consequences of Undervaccination - Measles Outbreak, New York City, 2018-2019. New England Journal of Medicine. Published online March 11, 2020. doi:10.1056/NEJMoa1912514

22. Bellafante G. Refuse to Vaccinate? Little Religious Ground to Stand On. The New York Times. https://www.nytimes.com/2015/02/15/nyregion/refuse-to-vaccinate-little-religious-ground-tostand-on.html. Published February 13, 2015. Accessed February 1, 2022.

23. Wincorn M. Schools Taking Stricter Approach as Measles Outbreak Continues in Lakewood Hamodia.com. Hamodia. Published November 13, 2018. Accessed February 2, 2022. https://hamodia.com/2018/11/13/schools-taking-stricter-approach-measles-outbreak-continueslakewood/

24. r. Fuerst S, r. Reiss Y. Beit Din Letter. Published November 15, 2018. Accessed February 2, 2022. https://images.hamodia.com/hamod-uploads/2018/11/15170939/ABC-1024x575.jpg

25. Der Yaid newspaper. Senseless! Heartless! Torah-less and Reckless. Published April 12, 2019. Accessed February 2, 2022. https://www.theyeshivaworld.com/wpcontent/uploads/2019/04/Editorial.pdf

26. Enman S. One Williamsburg school "ignited" NYC's measles crisis. Brooklyn Eagle. Published June 25, 2019. Accessed February 1, 2022. https://brooklyneagle.com/articles/2019/06/25/williamsburg-school-ignited-nyc-measles-crisis/

27. Borchardt R. As NYC Battles Measles Outbreak, Williamsburg Yeshivos Allege Over-Aggression Jewish Culture. Hamodia. Published June 30, 2019. Accessed February 1, 2022. https://hamodia.com/prime/nyc-battles-measles-outbreak-yeshivos-allege-aggression/

28. NNDSS. Annual statistics from the National Notifiable Diseases Surveillance System (NNDSS). Published February 4, 2022. Accessed February 4, 2022.

https://wonder.cdc.gov/nndss/nndss annual tables menu.asp 
29. Gold M. Parents Wanted Their Unvaccinated Children in School, but a Judge Said No. - The New York Times. Published March 13, 2019. Accessed February 1, 2022.

https://www.nytimes.com/2019/03/13/nyregion/measles-rockland-county.html

30. Gold M, Pager T. New York Suburb Declares Measles Emergency, Barring Unvaccinated Children From Public - The New York Times. Published March 26, 2019. Accessed February 1, 2022. https://www.nytimes.com/2019/03/26/nyregion/measles-outbreak-rockland-county.html

31. NYC DOHMH Oct 18 O. 2018 ALERT \#38: Measles Outbreak in New York City in the Orthodox Jewish Community. Published online October 18, 2018.

https://www1.nyc.gov/assets/doh/downloads/pdf/han/alert/2018/alert38-measles-outbreak.pdf

32. US News. New York Officials Ban Unvaccinated Children from School Amid Measles Outbreak. US News \& World Report. Published December 7, 2018. Accessed February 2, 2022. https://www.usnews.com/news/health-news/articles/2018-12-07/new-york-officials-banunvaccinated-children-from-school-amid-measles-outbreak

33. NYC DOHMH Letter. NEW YORK CITY DEPARTMENT OF HEALTH AND MENTAL HYGIENE. Published online December 6, 2018. https://www.jewishpress.com/wp-content/uploads/Measles-ExclusionPrincipals-Letter-Dec-6-2018-FINAL.pdf

34. NYC DOHMH Letter. NEW YORK CITY DEPARTMENT OF HEALTH AND MENTAL HYGIENE. Published online December 6, 2018. https://www.jewishpress.com/wp-content/uploads/Measles-ParentExcusion-Letter-Dec-6-2018-FINAL.pdf

35. NYC DOHMH Feb 28. 2019 ALERT \# 2: Update on Measles Outbreak in New York City in the Orthodox Jewish Community. Published online February 28, 2019. https://www1.nyc.gov/assets/doh/downloads/pdf/han/alert/2019/update-on-measles-outbreakin-nyc-in-the-orthodox-jewish-community.pdf

36. NYC DOHMH pr Mar 14. Yeshivas Allowing Unvaccinated Students to Attend School - NYC Health. Published March 14, 2019. Accessed January 22, 2022.

https://www1.nyc.gov/site/doh/about/press/pr2019/yeshivas-allowing-unvaccinated-students-toattend-school.page

37. NYC De Blasio. Transcript: De Blasio Administration Declares Public Health Emergency Due to Measles Crisis. Presented at: April 9, 2019. Accessed February 1, 2022.

http://www1.nyc.gov/office-of-the-mayor/news/188-19/transcript-de-blasio-administrationdeclares-public-health-emergency-due-measles-crisis

38. NYC DOHMH pr Apr 8. Commissioner Orders All Yeshivas to Exclude Unvaccinated Students - NYC Health. Published April 8, 2019. Accessed January 22, 2022.

https://www1.nyc.gov/site/doh/about/press/pr2019/commissioner-orders-all-yeshivas-toexclude-unvaccinated-students.page

39. NYC DOHMH Apr 9. ORDER OF THE COMMISSIONER. Published online April 9, 2019. https://www1.nyc.gov/assets/doh/downloads/pdf/press/2019/emergency-orders-measles 
40. AAPS. New York Threatens Prison for Vaccine Refusal. AAPS | Association of American Physicians and Surgeons. Published April 9, 2019. Accessed February 1, 2022. https://aapsonline.org/newyork-threatens-prison-for-vaccine-refusal//

41. NYC DOHMH Apr 17. NYC Board of Health. Published online April 17, 2019. https://web.archive.org/web/20190510172753/https://www1.nyc.gov/assets/doh/downloads/pd f/notice/2019/board-of-health-resolution-measles.pdf

42. CDC Archived. Measles - NYC Health. Published April 19, 2019. Accessed February 1, 2022. https://web.archive.org/web/20190419021905/https:/www1.nyc.gov/site/doh/health/healthtopics/measles.page

43. NYC DOHMH Apr 24. 2019 ALERT \# 9: Citywide Recommendations during the Ongoing Measles Outbreak in New York City. Published online April 24, 2019.

https://www1.nyc.gov/assets/doh/downloads/pdf/han/alert/2019/recommendations-duringmeasles-outbreak.pdf

44. NYC DOHMH Nov 5. 2018 ALERT \#39: Update on Measles Outbreak in New York City in the Orthodox Jewish Community. Published online November 5, 2018. https://www1.nyc.gov/assets/doh/downloads/pdf/han/alert/2018/alert39-measles-outbreak.pdf

45. Rosen J. measles in nyc presentation for providers. Presented at: June 12, 2019. Accessed February 1, 2022. https://www1.nyc.gov/assets/doh/downloads/pdf/imm/measles-in-nyc-presentation-forproviders

46. NYC DOHMH May 24. 2019 ALERT \#10: Update on Measles Outbreak in New York City and Citywide Recommendations. Published online May 24, 2019. https://www1.nyc.gov/assets/doh/downloads/pdf/han/alert/2019/update-measles-outbreak.pdf

47. NYC DOHMH Sep 3. 2019 ALERT \#26: Update on Measles Outbreak in New York City. Published online September 3, 2019. https://www1.nyc.gov/assets/doh/downloads/pdf/han/alert/2019/measles-transmissionended.pdf

48. Gastañaduy PA, Budd J, Fisher N, et al. A Measles Outbreak in an Underimmunized Amish Community in Ohio. N Engl J Med. 2016;375(14):1343-1354. doi:10.1056/NEJMoa1602295

49. Banerjee E, Griffith J, Kenyon C, et al. Containing a measles outbreak in Minnesota, 2017: methods and challenges. Perspect Public Health. 2020;140(3):162-171. doi:10.1177/1757913919871072

50. Minnesota DoH. Measles, 2017: DCN - Minnesota. Published 2017. Accessed January 31, 2022. https://www.health.state.mn.us/diseases/reportable/dcn/sum17/measles.html

51. Hall V, Banerjee E, Kenyon C, et al. Measles Outbreak - Minnesota April-May 2017. MMWR Morb Mortal Wkly Rep. 2017;66(27):713-717. doi:10.15585/mmwr.mm6627a1

52. Stein-Zamir C, Abramson N, Shoob H. Large Measles Outbreak in Orthodox Jewish Communities Jerusalem District, Israel, 2018-2019. MMWR Morb Mortal Wkly Rep. 2020;69(18):562-563. doi:10.15585/mmwr.mm6918a3 
501

502

503

504

505

506

507

508
53. Krakow RJ. CHD v. NYC DOHMH. Published online April 15, 2019. Accessed February 2, 2022. https://childrenshealthdefense.org/wp-content/uploads/04-15-19-CF-et-al-v-CF-et-alAFFIRMATION 2.pdf

54. NYS DOH. School Immunization Survey: Beginning 2012-13 School Year | State of New York. New York State Department of Health | Health Data NY. Published February 7, 2022. Accessed February 7, 2022. https://health.data.ny.gov/Health/School-Immunization-Survey-Beginning-2012-13Schoo/5pme-xbs5 